\title{
Functional role of miR-27b in the development of gastric cancer
}

\author{
XIAOJIE CHEN $^{1 *}$, YEJIA CUI $^{2 *}$, XUHONG XIE $^{1}$, YANFEN XING $^{1}$, ZHAOHU YUAN $^{1}$ and YAMING WEI \\ ${ }^{1}$ Department of Blood Transfusion, Guangzhou First People's Hospital, Guangzhou Medical University, \\ Guangzhou, Guangdong 510180; ${ }^{2}$ Department of Clinical Laboratory, Dongguan \\ Third People's Hospital, Dongguan, Guangdong 523326, P.R. China
}

Received November 17, 2016; Accepted July 7, 2017

DOI: $10.3892 / \mathrm{mmr} .2018 .8538$

\begin{abstract}
Previous studies have demonstrated that microRNAs (miRNAs/miRs) act as tumor suppressors or oncogenes during multiple processes in cancer. It has been observed that miR-27b may act as a tumor-suppressor and was significantly downregulated in a number of types of cancer. However, the functions of miR-27b in gastric cancer (GC) remain unclear. The present study aimed to investigate the functional role of miR-27b in the progression of GC. The downregulation of miR-27b in human GC plasma was confirmed using miRNA microarray and reverse transcription-quantitative polymerase chain reaction analyses. The association between circulating miR-27b expression and clinicopathological features of GC was analyzed and the results demonstrated that the level of circulating miR-27b was significantly correlated with GC differentiation. Receiver operating characteristic curve analysis identified that the plasma level of miR-27b may be a potential biomarker for differentiating patients with $\mathrm{GC}$ from healthy controls. In order to investigate the effect of miR-27b on GC cell behavior, miR-27b was overexpressed using miR-27b mimics, and it was observed that miR-27b was able to inhibit cell proliferation and induce apoptosis in SGC7901 cells. Previous studies have demonstrated that vascular endothelial growth factor $\mathrm{C}$ (VEGFC) is a target of miR-27b, and the results of the present study were consistent with these reports. Taken together, the results of the present study indicated that miR-27b may act as a potential biomarker for differentiating patients with GC from healthy controls, and serve as a tumor suppressor in GC by targeting VEGFC.
\end{abstract}

Correspondence to: Dr Yaming Wei, Department of Blood Transfusion, Guangzhou First People's Hospital, Guangzhou Medical University, 1 Panfu Road, Guangzhou, Guangdong 510180, P.R. China

E-mail: weiym1234@sina.com

*Contributed equally

Key words: microRNA-27b, gastric cancer, receiver operating characteristic curve, vascular endothelial growth factor $\mathrm{C}$, proliferation, apoptosis

\section{Introduction}

Gastric cancer (GC) is the fourth most prevalent type of malignancy and the second leading cause of cancer-associated mortality worldwide; GC exhibits its highest incidence rates in East Asia, Eastern Europe, and South America (1). Over 70\% of new cases and mortalities occur in developing countries (1). The 5-year survival rate for patients with GC is $<20 \%$ due to the frequently late diagnosis; when the tumor is diagnosed and treated at an early stage, the 5-year survival rate may reach $90 \%$ (2). The majority of cases of GC are asymptomatic or cause nonspecific symptoms, which may lead to a delayed diagnosis. Approximately $80 \%$ of patients are diagnosed at the advanced stages in the majority of countries (3). The conventional treatments for advanced GC, including chemotherapy, radiotherapy and surgery, are associated with poor outcomes (4). Therefore, it is necessary to develop novel noninvasive biomarkers to improve early prognostic prediction, and to develop more effective treatment strategies for GC.

MicroRNAs (miRNAs/miRs) are 19-22-base small non-coding RNA molecules that regulate protein-coding gene expression via the degradation of target mRNAs, or repress translation by binding to the 3 '-untranslated regions (3'-UTRs) of the target mRNAs (5). Circulating miRNAs are stable under extreme conditions, including boiling, low or high $\mathrm{pH}$, extended storage and freeze-thaw cycles (6), indicating that they may serve as noninvasive biomarkers for the diagnosis of cancer and other diseases. Accumulating evidence has demonstrated that miRNAs are involved in various cellular processes, including proliferation, apoptosis, development, differentiation and metabolism (7). miRNAs function as oncogenes or tumor suppressors and are aberrantly expressed in various types of malignancy, including pancreatic cancer (8) and GC (9).

Previous studies have demonstrated that miR-23b/27b clustered miRNAs were markedly reduced in numerous types of cancer (10-12). However, the potential role of miR-27b in GC remains unclear. Therefore, the aim of the present study was to determine the functional roles served by miR-27b in the development of GC and to elucidate the molecular mechanisms underlying the pathogenesis of GC.

\section{Materials and methods}

Patients and plasma samples. Plasma samples from 46 patients (31 males and 15 females; age, 31-76 years) with GC who had 
not received surgery, chemotherapy or radiotherapy prior to blood sample collection, and 40 healthy controls (22 males and 18 females; age, 38-70 years), were obtained between January and October 2015 at Guangzhou First People's Hospital of Guangzhou Medical University (Guangzhou,China). The present study was approved by the ethics committee of Guangzhou First People's Hospital of Guangzhou Medical University and informed consent was obtained from each patient.

miRNA microarray analysis. Plasma from 5 random patients with GC and 3 healthy controls was used for miRNA microarray analysis (KangChen Bio-tech, Inc., Shanghai, China). Total RNA was isolated using an miRNeasy mini kit (Qiagen $\mathrm{GmbH}$, Hilden, Germany), according to the manufacturer's protocol. The samples were labeled using the miRCURY ${ }^{\mathrm{TM}} \mathrm{Hy} 3^{\mathrm{TM}} / \mathrm{Hy} 5^{\mathrm{TM}}$ Power labeling kit and hybridized on the miRCURY ${ }^{\mathrm{TM}}$ LNA microRNA Array (version 18.0; Exiqon, Inc., Vedbaek, Denmark). Following salt buffer and detergent (included in the miRCURY ${ }^{\mathrm{TM}} \mathrm{Hy}^{\mathrm{TM}} / \mathrm{Hy}^{\mathrm{TM}}$ Power labeling kit; Exiqon, Inc.) washing steps, and in situ hybridization, the slides were scanned using the Axon GenePix 4000B microarray scanner (Molecular Devices LLC, Sunnyvale, CA, USA). Scanned images were then imported into GenePix Pro 6.0 software (Molecular Devices LLC) for grid alignment and data extraction.

Cell culture and transfection. The human GC cell line SGC7901 was purchased from Nanjing KeyGen Biotech Co., Ltd. (Nanjing, China). The cells were cultured in RPMI-1640 medium (Gibco; Thermo Fisher Scientific, Inc., Waltham, MA, USA) containing 10\% fetal bovine serum (Gibco; Thermo Fisher Scientific, Inc.) at $37^{\circ} \mathrm{C}$ in a humidified atmosphere with $5 \% \mathrm{CO}_{2}$. SGC7901 cells $\left(5 \times 10^{4}\right.$ cells $\left./ \mathrm{ml}\right)$ were transfected with the miR-27b mimic (miR-27b-3p mimic sequence 5'-UUC ACAGUGGCUAAGUUCUGC-3') or its negative control (5'-UUUGUACUACACAAAAGUACUG-3'; both Guangzhou RiboBio Co., Ltd., Guangzhou, China) using Lipofectamine 2000 (Invitrogen; Thermo Fisher Scientific, Inc.), according to the manufacturer's protocol, at a final concentration of $50 \mathrm{nM}$. Following $24 \mathrm{~h}$ culture, transfection efficiency was monitored using reverse transcription-quantitative polymerase chain reaction (RT-qPCR) analysis.

RNA isolation and RT-qPCR analysis. Total RNA was extracted from SGC7901 cells using TRIzol reagent (Invitrogen; Thermo Fisher Scientific, Inc.), according to the manufacturer's protocol. Reverse transcription was performed with a reaction mixture of: $5 \mathrm{x}$ reaction buffer $(2.0 \mu \mathrm{l})$, nuclease-free water $(5.0 \mu \mathrm{l})$, enzyme mix Template $(1.0 \mu \mathrm{l})$ and total RNA $(2.0 \mu \mathrm{l})$. The reaction mixture was incubated for $60 \mathrm{~min}$ at $42^{\circ} \mathrm{C}$, heat-inactivated for $5 \mathrm{~min}$ at $95^{\circ} \mathrm{C}$ prior to immediate cooling at $4^{\circ} \mathrm{C}$. The expression level of miR-27b-3p [mature sequence, 5'-UUCACAGUG GCUAAGUUCUGC-3' (primer sequence unavailable); Takara Biotechnology Co., Ltd., Dalian, China] was assessed using miRCURY LNA ${ }^{\mathrm{TM}} \mathrm{SYBR}^{\circledR}$ Green master mix (Exiqon, Inc.). miR-16 [mature sequence, 5'-UAGCAGCACGUAAAUAUU GGCG-3' (primer sequence unavailable); Takara Biotechnology Co., Ltd.] was used for normalization. The qPCR reaction system contained: SYBR-Green master mix $(4.8 \mu \mathrm{l})$, PCR primer mix (1.0 $\mu \mathrm{l})$, diluted cDNA templates $(4.0 \mu \mathrm{l})$, ROX Reference Dye II $(0.2 \mu \mathrm{l})$. PCR was performed with the following thermocycling conditions: $95^{\circ} \mathrm{C}$ for $10 \mathrm{~min}$, followed by 40 cycles of $95^{\circ} \mathrm{C}$ for $10 \mathrm{sec}$ and $60^{\circ} \mathrm{C}$ for $60 \mathrm{sec}$.

For quantitative analysis of vascular endothelial growth factor C (VEGFC) mRNA expression, a RT-PCR kit (Takara Biotechnology Co., Ltd.) was used to amplify the target genes, according to the manufacturer's protocol. The expression level of VEGFC was normalized using $\beta$-actin mRNA levels. $\beta$-actin and VEGFC for RT-qPCR were synthesized by Takara Biotechnology Co., Ltd. The primer sequences were as follows: $\beta$-actin, forward (5'-3') GTAAAGACCTCTATGCCA ACA and reverse (5'-3') GGACTCATCGTACTCCTGCT; VEGFC, forward (5'-3') AGCACGAGCTACCTCAGCAAG AC and reverse (5'-3') TTTAGACATGCATCGGCAGGAA. The relative expression levels were calculated using the comparative $2^{-\Delta \Delta \mathrm{Cq}}$ method (13).

Cell proliferation assays. SGC7901 cells were seeded in 96 -well plates $\left(5 \times 10^{3}\right.$ cells/well). Following culture at $37^{\circ} \mathrm{C}$ for 24, 48 and $72 \mathrm{~h}$, respectively, an MTS assay was performed. A total of $20 \mu \mathrm{l}$ reagent (CellTiter 96 AQueous One Solution Reagent; Promega Corporation, Madison, WI, USA) was added into each well and incubated for $4 \mathrm{~h}$ at $37^{\circ} \mathrm{C}$ in a humidified atmosphere with $5 \% \mathrm{CO}_{2}$. The absorbance was recorded at $490 \mathrm{~nm}$ using a 96-well plate reader. The proliferation analysis experiments were performed in triplicate.

Cellular apoptosis analysis. SGC7901 cells were seeded in 6 -well plates at a density of $10^{5}$ cells/well. A total of $24 \mathrm{~h}$ subsequent to transfection, SGC7901 cells were stained with Annexin V-fluorescein isothiocyanate and propidium iodide (Bestbio, Shanghai, China), according to the manufacturer's protocol. Apoptosis rates were analyzed using Guava EasyCyte Mini System with Cytosoft version 1.2 (EMD Millipore, Billerica, MA, USA). The experiments were repeated three times.

Statistical analysis. All statistical analyses were performed using SPSS 13.0 software (SPSS, Inc., Chicago, IL, USA). The Mann-Whitney $U$ test was used to compare the differences in plasma miR-27b level between the patients with GC and the healthy controls. A receiver operating characteristic (ROC) curve and the area under the ROC curve was used to assess the utility of miR-27b expression levels in distinguishing patients with GC from the healthy controls. All values were presented as the mean \pm standard deviation. Statistical analysis was performed using one-way analysis of variance, differences among specific means were assessed by the least significant difference post hoc test. $\mathrm{P}<0.05$ was considered to indicate a statistically significant difference.

\section{Results}

miRNAs are expressed differentially between patients with $G C$ and healthy controls. miRNA microarray profiling was performed in $5 \mathrm{GC}$ and 3 healthy control plasma samples. Hierarchical clustering analysis based on the miRNA expression pattern indicated a significant difference between GC plasma and the matched healthy controls. It was observed that 17 miRNAs were significantly upregulated in GC plasma and 64 miRNAs were downregulated compared with the healthy controls (Fig. 1). 


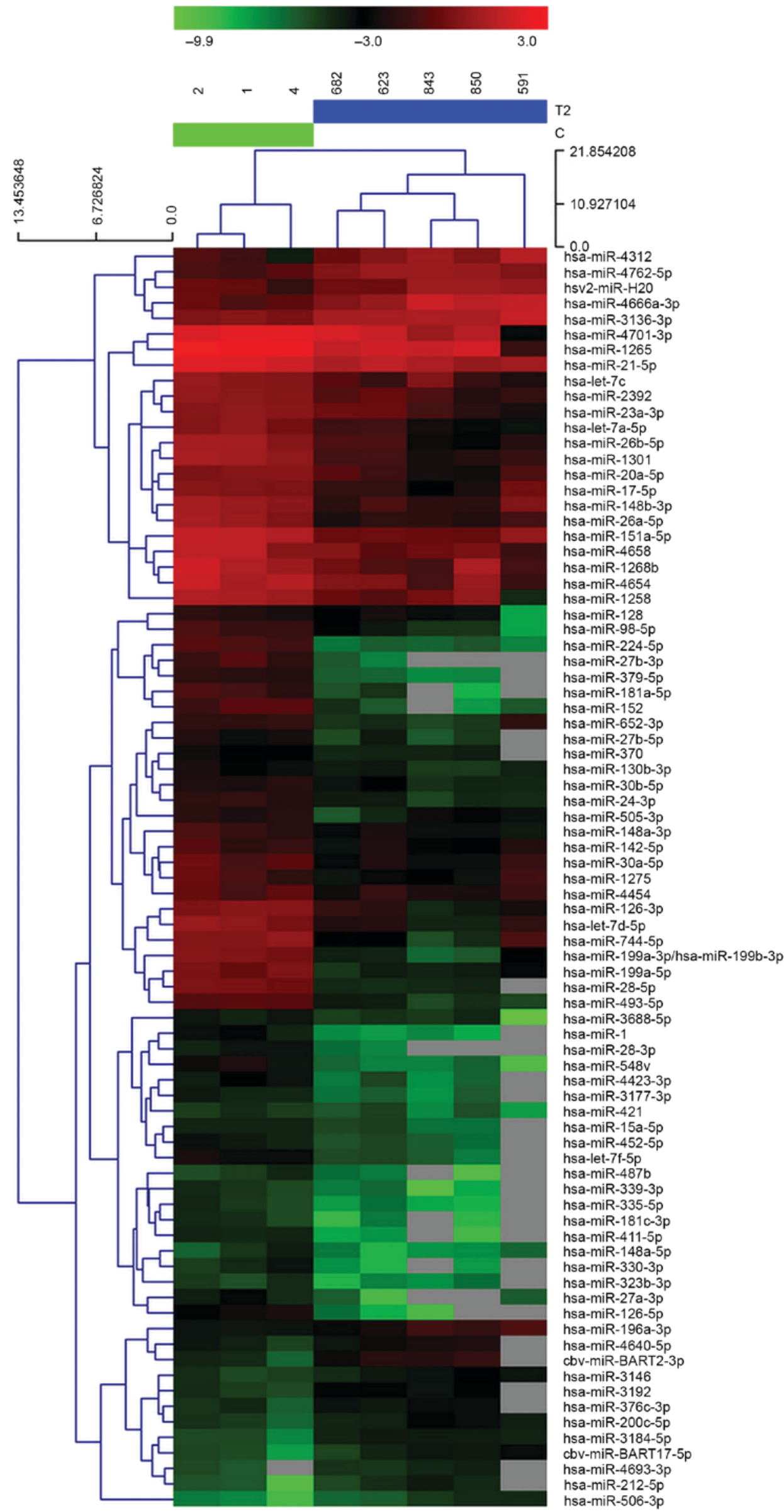

Figure 1. Hierarchical clustering of total miRNAs in the raw data from 5 patients with gastric cancer and 3 healthy controls. Rows, expressed miRNAs; columns, sample numbers. Red color represents upregulation and green color represents downregulation of the expression of the miRNAs. miRNA, microRNA. 
Table I. The association between plasma miR-27b-3p expression and clinicopathological characteristics in patients with gastric cancer $(n=46)$.

\begin{tabular}{|c|c|c|c|c|}
\hline Clinicopathological features & No. cases $(\%)$ & miR-27b-3p level $\left(2^{-\Delta \Delta C q}\right)$ & $\mathrm{U}$ & P-value \\
\hline Gender & & & 167.0 & 0.125 \\
\hline Male & $31(67.4)$ & $1.620 \times 10^{-2}\left(8.900 \times 10^{-3}, 3.960 \times 10^{-2}\right)$ & & \\
\hline Female & $15(32.6)$ & $3.880 \times 10^{-2}\left(8.000 \times 10^{-3}, 9.390 \times 10^{-2}\right)$ & & \\
\hline Tumor location & & & 260.0 & 0.930 \\
\hline Body + cardia & $22(47.8)$ & $2.060 \times 10^{-2}\left(9.700 \times 10^{-3}, 4.710 \times 10^{-2}\right)$ & & \\
\hline Antrum & $24(52.2)$ & $2.070 \times 10^{-2}\left(7.900 \times 10^{-3}, 6.180 \times 10^{-2}\right)$ & & \\
\hline TNM stage & & & 249.0 & 0.733 \\
\hline $\mathrm{I}+\mathrm{II}$ & $23(50.0)$ & $1.490 \times 10^{-2}\left(8.800 \times 10^{-3}, 6.000 \times 10^{-2}\right)$ & & \\
\hline III+IV & $23(50.0)$ & $2.450 \times 10^{-2}\left(8.900 \times 10^{-3}, 4.730 \times 10^{-2}\right)$ & & \\
\hline Differentiation & & & 155.0 & $0.029^{\mathrm{a}}$ \\
\hline Poor & $28(60.9)$ & $1.460 \times 10^{-2}\left(7.800 \times 10^{-3}, 3.670 \times 10^{-2}\right)$ & & \\
\hline Well and moderate & $18(39.1)$ & $3.920 \times 10^{-2}\left(1.200 \times 10^{-2}, 7.600 \times 10^{-2}\right)$ & & \\
\hline Regional lymph node metastasis & & & 255.0 & 0.869 \\
\hline Yes & $25(54.3)$ & $2.370 \times 10^{-2}\left(9.100 \times 10^{-3}, 4.350 \times 10^{-2}\right)$ & & \\
\hline No & $21(45.7)$ & $1.760 \times 10^{-2}\left(7.500 \times 10^{-3}, 6.060 \times 10^{-2}\right)$ & & \\
\hline Metastasis & & & 235.0 & 0.559 \\
\hline Yes & $11(63.4)$ & $1.760 \times 10^{-2}\left(8.900 \times 10^{-3}, 6.120 \times 10^{-2}\right)$ & & \\
\hline No & $35(36.6)$ & $2.370 \times 10^{-2}\left(3.300 \times 10^{-3}, 3.930 \times 10^{-2}\right)$ & & \\
\hline
\end{tabular}

${ }^{\mathrm{a}} \mathrm{P}<0.05 . \mathrm{miR}$, microRNA.

Expression of miR-27b is downregulated in GC plasma. To verify the microarray data, RT-qPCR was used to compare the expression of miR-27b between 46 samples of GC plasma and 40 samples of healthy control plasma. miR-16 expression was used as an internal reference to normalize the RT-qPCR data (14). As presented in Fig. 2, it was observed that the expression level of miR-27b was significantly decreased in GC compared with healthy controls. The results of the microarray analysis were consistent with those from RT-qPCR.

Plasma miR-27b correlates with clinicopathological features in $G C$. The association between circulating miR-27b expression and clinicopathological features of GC is summarized in Table I. The results demonstrated that the level of circulating miR-27b was significantly correlated with differentiation $(\mathrm{P}=0.029)$. However, there was no correlation of miR-27b expression with other clinical features, including age, gender, tumor size and invasion (all $\mathrm{P}>0.05$ ).

Diagnostic value of circulating $m i R-27 b$. ROC curve analysis indicated that the plasma level of miR-27b is a potential biomarker for differentiating patients with GC from healthy controls, with a ROC curve area of 0.724 (95\% confidence interval=0.618-0.831; $\mathrm{P}=0.0004$ ) (Fig. 3). When the threshold value for miR-27b was 0.0495 , the sensitivity was $76.1 \%$ with a specificity of $60 \%$.

Overexpression of $m i R-27 b$ was examined by $R T-q P C R$ analysis. In order to investigate the role of miR-27b in GC cells, miR-27b was overexpressed using miR-27b mimics,

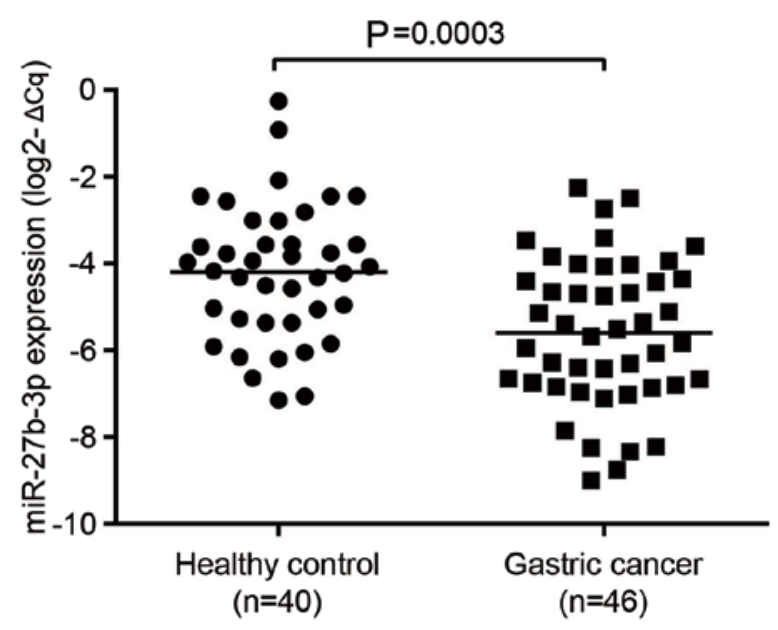

Figure 2. Plasma miR-27b-3p levels from gastric cancers and healthy controls were detected with reverse transcription-quantitative polymerase chain reaction analysis. The expression level of miR-27b-3p was significantly decreased in gastric cancer $(n=46)$ compared with healthy control samples $(\mathrm{n}=40 ; \mathrm{P}=0.0003)$. miR, microRNA.

and the expression of miR-27b was determined by RT-qPCR analysis. The results of the present study demonstrated that the expression of miR-27b in the blank control, negative control and miR-27b mimics groups were $0.022 \pm 0.104,0.016 \pm 0.005$ and $1.493 \pm 0.179$, respectively. There were $\sim 67.87$-fold and 91.60-fold increases in miR-27b levels in SGC7901 cells transfected with miR-27b mimics compared with the blank control and the negative control, respectively ( $\mathrm{P}<0.05$; Fig. 4). 


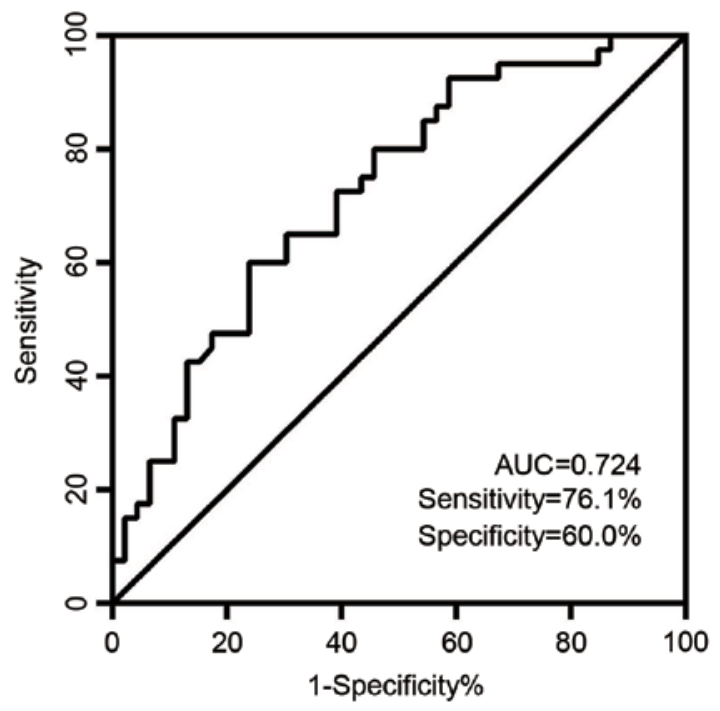

Figure 3. Receiver operating characteristic curve analysis of plasma miR-27b-3p. miR-27b-3p yielded an AUC of 0.724 with $76.1 \%$ sensitivity and $60.0 \%$ specificity in discriminating between gastric cancer and healthy control plasma samples. miR, microRNA; AUC, area under the curve.

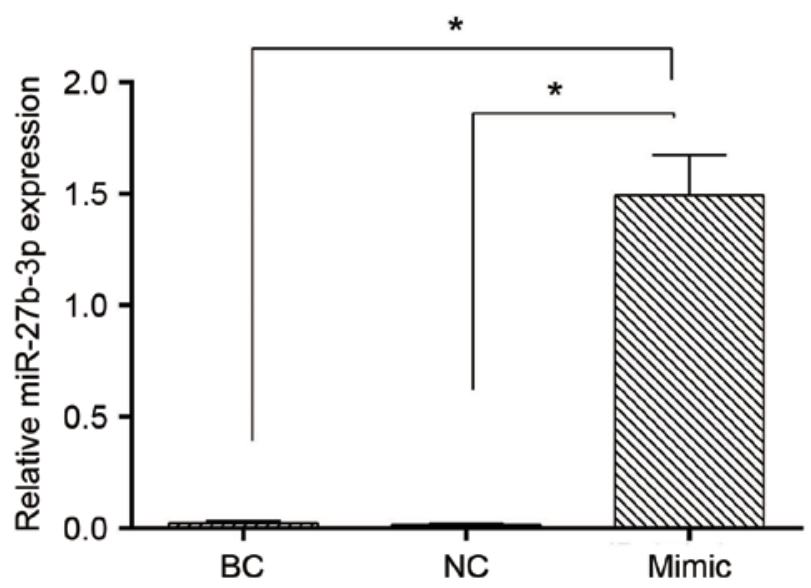

Figure 4. Expression of miR-27b in SGC7901 cells transfected with miR-27b mimics. miR-27b was overexpressed using miR-27b mimics, and the expression of miR-27b was determined by reverse transcription-quantitative polymerase chain reaction analysis. ${ }^{*} \mathrm{P}<0.05$. BC, blank control; $\mathrm{NC}$, negative control; miR, microRNA.

miR-27b overexpression induces GC cellular apoptosis. In order to evaluate the tumor-suppressing potential of miR-27b, SGC7901 cells were transiently transfected with miR-27b mimics or negative control miRNA. Flow cytometric analysis demonstrated that apoptosis in SGC7901 cells transfected with miR-27b mimics was significantly increased compared with cells transfected with the negative control or untreated cells (Fig. 5), suggesting that miR-27b may induce cellular apoptosis. These results indicated that miR-27b may function as a tumor suppressor in human GC development by inducing apoptosis.

miR-27b overexpression suppresses GC cell proliferation. In order to evaluate the effects of miR-27b overexpression on cell proliferation, miR-27b mimics or negative control were transfected into SGC7901 cells. An MTS assay demonstrated that miR-27b mimics induced a significant inhibition of cell proliferation compared with the negative control and blank control on the 3rd day (Fig. 6; $\mathrm{P}<0.05$ ). Furthermore, a cell growth curve assay additionally demonstrated that miR-27b mimics inhibited cell growth (Fig. 6).

miR-27b inhibits the expression of VEGFC. Previous studies have demonstrated that VEGFC is a target of miR-27b and it can be inhibited by miR-27b $(15,16)$. In order to confirm that miR-27b represses VEGFC expression in SGC7901 cells, RT-qPCR analysis was performed in the present study, and it was observed that transfection with miR-27b mimics led to a significant decrease in VEGFC level ( $\mathrm{P}<0.05$; Fig. 7).

\section{Discussion}

GC is a lethal malignancy worldwide (17). Due to the nonspecific symptoms of the early stages of GC, diagnosis is frequently delayed. The 5-year survival rate of GC is $<20 \%$ (12). Therefore, it is necessary to understand the underlying molecular mechanisms and progression stages of the disease, and to investigate novel therapeutic targets.

Numerous studies have demonstrated that the abnormal expression of miRNAs serve a pivotal role in carcinogenesis and cancer progression $(10-12,18)$. Circulating miRNAs are able to withstand harsh conditions (6). Therefore, miRNAs may serve as predictive biomarkers and therapeutic targets for GC.

miR-27b located on chromosome 19p13 which is belongs to the $\mathrm{miR}-23 \mathrm{~b} / \mathrm{miR}-27 \mathrm{~b}$ cluster (14). miR-27b has been reported to act as a tumor suppressor in certain types of human malignancy, including neuroblastoma (17), clear cell renal cell carcinoma (18), pancreatic cancer (19) and lung cancer (20).

In the present study, it was demonstrated that miR-27b expression was significantly downregulated in the plasma of patients with GC, and it was demonstrated that decreased expression of miR-27b was associated with poor differentiation. Overexpression of miR-27b inhibited cancer cell proliferation and induced apoptosis in SGC7901 cells. VEGFC belongs to the platelet-derived growth factor family, and is a potent inducer of lymphangiogenesis by activating the vascular endothelial growth factor receptor 3 signaling pathway (21). The present study confirmed that VEGFC may be inhibited by miR-27b. The results of the present study demonstrated that miR-27b may be a candidate tumor suppressor by inhibiting VEGFC in GC. Previous studies have demonstrated that VEGFC is a target of miR-27b $(15,16)$. miR-27b was observed to be downregulated in colorectal cancer, and overexpression of miR-27b repressed colorectal cancer cell proliferation, colony formation and tumor growth by targeting VEGFC (17). Downregulation of VEGFC in lung and colon cancer cells led to a significant inhibition of tumor growth and metastasis (18). VEGFC is therefore an attractive target for the development of novel anticancer strategies.

Overexpression of VEGFA and VEGFC in GC has been demonstrated to correlate with prognosis, and their silencing may effectively inhibit cancer growth (22). VEGFC was highly expressed in the gastric tumor tissues obtained in the present study. Additionally, the expression of VEGFC was correlated with the pathological staging of GC. The growth of gastric tumor cells was significantly inhibited by transfecting with an antisense VEGFC gene (23). Various studies have 
$\mathrm{BC}$

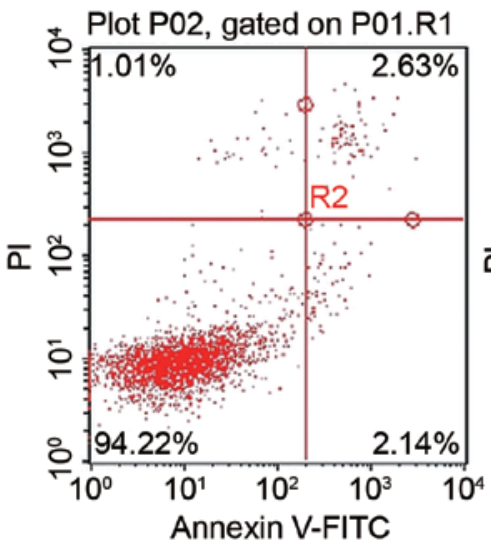

$\mathrm{NC}$

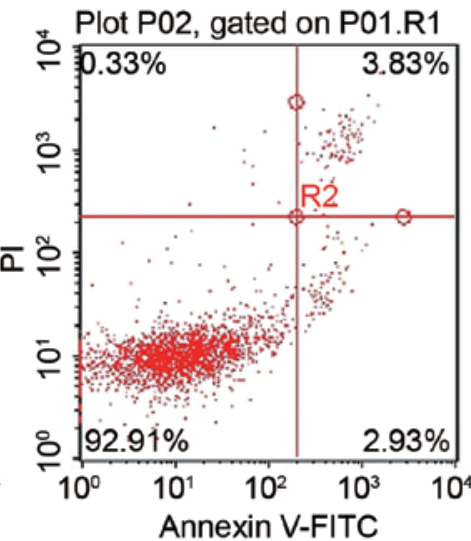

Mimic

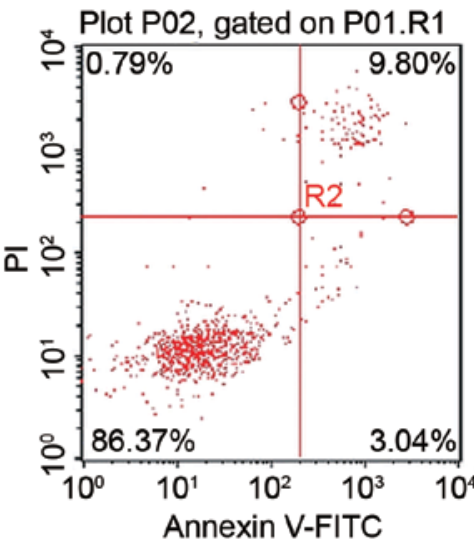

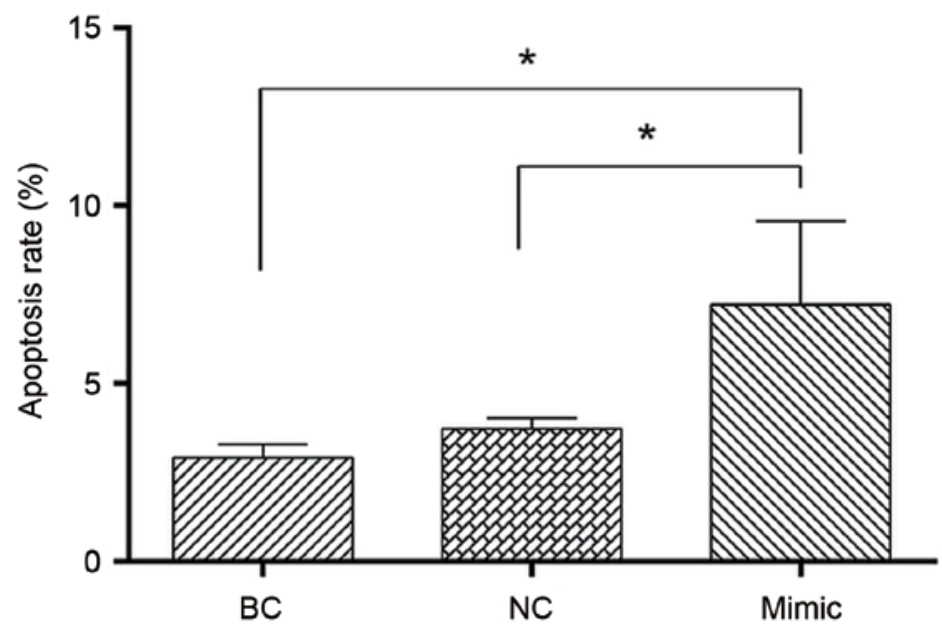

Figure 5. miR-27b induces apoptosis in SGC7901 cells. Flow cytometric analysis demonstrated that overexpression of miR-27b significantly induced apoptosis. Cellular apoptosis was evaluated using Annexin V-FITC/PI double staining. "P<0.05. BC, blank control; NC, negative control; miR, microRNA; FITC, fluorescein isothiocyanate; PI, propidium iodide.

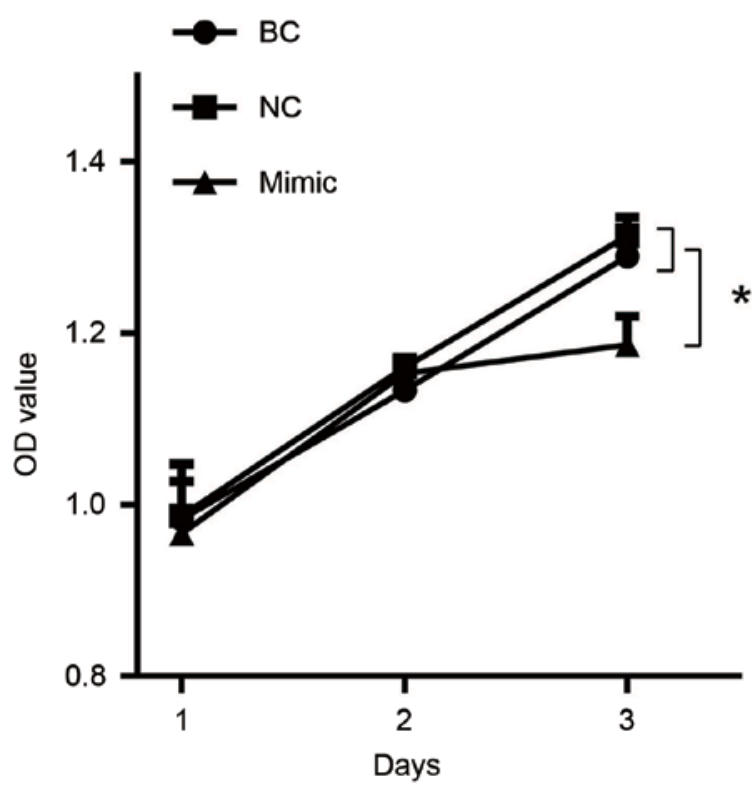

Figure 6. Effects of miR-27b on the proliferation of SGC-7901 cells Overexpression of miR-27b was achieved by transfecting miR-27b mimics. SGC7901 cells transfected with negative control and without any treatment (blank control) were used as controls. An MTS assay was performed to measure cell proliferation. $\mathrm{n}=3$. ${ }^{*} \mathrm{P}<0.05$. $\mathrm{BC}$, blank control; NC, negative control; miR, microRNA; OD, optical density.

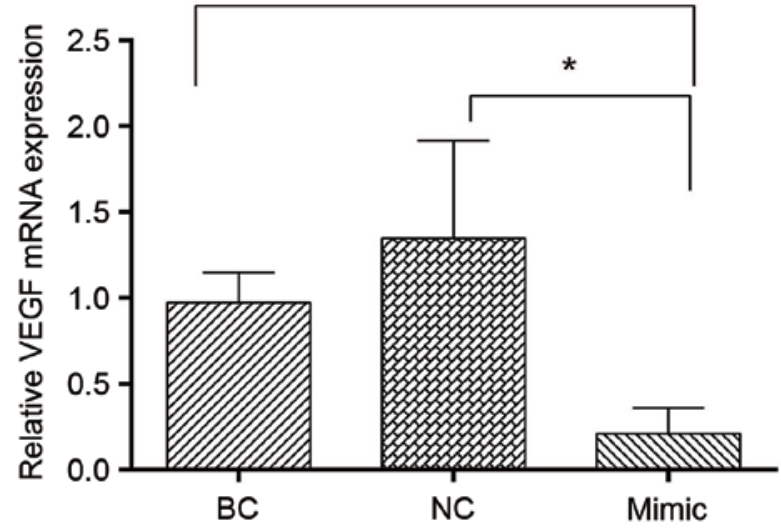

Figure 7. miR-27b represses VEGFC expression in SGC7901 cells. Reverse transcription-quantitative polymerase chain reaction analysis of the VEGFC mRNA expression level in SGC7901 cells. $\mathrm{n}=3$. ${ }^{*} \mathrm{P}<0.05$. BC, blank control; $\mathrm{NC}$, negative control; miR, microRNA; VEGFC, vascular endothelial growth factor $\mathrm{C}$.

demonstrated VEGFC can induce lymphangiogenesis (24-26). Overexpression of VEGFC has been observed to increase leukemic cell proliferation in a dose-dependent manner by 
inducing the expression of apoptosis regulator $\mathrm{Bcl}-2$ and increasing the Bcl-2/apoptosis regulator Bax ratio (27).

In the present study, it was observed that miR-27b significantly decreased VEGFC mRNA expression, inhibited cancer cell proliferation and induced apoptosis in SGC7901 cells. Therefore, it is possible that VEGFC is associated with lymphangiogenesis in addition to cell proliferation and apoptosis.

In conclusion, the results of the present study demonstrated that plasma miR-27b was downregulated in GC. The plasma level of miR-27b is a potential biomarker for the differentiation between patients with GC and healthy individuals. Upregulation of miR-27b suppressed cell proliferation and induced apoptosis in GC cell lines. Blocking the VEGFC signaling pathway may prove to be an effective treatment for GC by targeting GC cells in addition to the neovasculature. The results of the present study suggested that miR-27b may be a potential biomarker and molecular therapeutic target for $\mathrm{GC}$.

\section{Acknowledgements}

The present study was supported by the Guangzhou Key Project of Medical and Health Science and Technology (grant no. 201102A212011).

\section{Competing interests}

The authors declare they have no competing interests.

\section{References}

1. Jemal A, Bray F, Center MM, Ferlay J, Ward E and Forman D Global cancer statistics. CA Cancer J Clin 61: 69-90, 2011.

2. Miyahara R, Niwa Y, Matsuura T, Maeda O, Ando T, Ohmiya N, Itoh A, Hirooka Y and Goto H: Prevalence and prognosis of gastric cancer detected by screening in a large Japanese population: Data from a single institute over 30 years. J Gastroenterol Hepatol 22: 1435-1442, 2007.

3. Correa P: Gastric cancer: Overview. Gastroenterol Clin North Am 42: 211-217, 2013

4. Takahashi T, Saikawa Y and Kitagawa Y: Gastric cancer: Current status of diagnosis and treatment. Cancers (Basel) 5: 48-63, 2013

5. Bartel DP: MicroRNAs: Genomics, biogenesis, mechanism, and function. Cell 116: 281-297, 2004.

6. Chen X, Ba Y, Ma L, Cai X, Yin Y, Wang K, Guo J, Zhang Y, Chen J, Guo X, et al: Characterization of microRNAs in serum: A novel class of biomarkers for diagnosis of cancer and other diseases. Cell Res 18: 997-1006, 2008.

7. Ambros V: The functions of animal microRNAs. Nature 431: 350-355, 2004

8. Hamada S, Satoh K, Fujibuchi W, Hirota M, Kanno A, Unno J, Masamune A, Kikuta K, Kume K and Shimosegawa T: miR-126 acts as a tumor suppressor in pancreatic cancer cells via the regulation of ADAM9. Mol Cancer Res 10: 3-10, 2012.

9. Hashiguchi Y, Nishida N, Mimori K, Sudo T, Tanaka F, Shibata K, Ishii H, Mochizuki H, Hase K, Doki Y and Mori M: Down-regulation of miR-125a-3p in human gastric cancer and its clinicopathological significance. Int J Oncol 40: 1477-1482, 2012.

10. Chen L, Li H, Han L, Zhang K, Wang G, Wang Y, Liu Y, Zheng Y, Jiang T, Pu P, et al: Expression and function of miR-27b in human glioma. Oncol Rep 26: 1617-1621, 2011.
11. Lee JJ, Drakaki A, Iliopoulos D and Struhl K: miR-27b targets PPAR $\gamma$ to inhibit growth, tumor progression and the inflammatory response in neuroblastoma cells. Oncogene 31: 3818-3825, 2012.

12. Ishihara $T$, Seki $N$, Inoguchi $S$, Yoshino $H$, Tatarano $S$, Yamada $Y$, Itesako T, Goto Y, Nishikawa R, Nakagawa M and Enokida $\mathrm{H}$ : Expression of the tumor suppressive miRNA-23b/27b cluster is a good prognostic marker in clear cell renal cell carcinoma. J Urol 192: 1822-1830, 2014.

13. Schmittgen TD and Livak KJ: Analyzing real-time PCR data by the comparative C(T) method. Nat Protoc 3: 1101-1108, 2008.

14. Song J, Bai Z, Han W, Zhang J, Meng H, Bi J, Ma X, Han S and Zhang Z: Identification of suitable reference genes for qPCR analysis of serum microRNA in gastric cancer patients. Dig Dis Sci 57: 897-904, 2012.

15. Ye J, Wu X, Wu D, Wu P, Ni C, Zhang Z, Chen Z, Qiu F, Xu J and Huang J: miRNA-27b targets vascular endothelial growth factor $\mathrm{C}$ to inhibit tumor progression and angiogenesis in colorectal cancer. PLoS One 8: e60687, 2013.

16. Khromova N, Kopnin P, Rybko V and Kopnin BP: Downregulation of VEGF-C expression in lung and colon cancer cells decelerates tumor growth and inhibits metastasis via multiple mechanisms. Oncogene 31: 1389-1397, 2012.

17. Kamangar F,Dores GM and Anderson WF: Patterns of cancer incidence, mortality, and prevalence across five continents: Defining priorities to reduce cancer disparities in different geographic regions of the world. J Clin Oncol 24: 2137-2150, 2006.

18. Lu J, Getz G, Miska EA, Alvarez-Saavedra E, Lamb J, Peck D, Sweet-Cordero A, Ebert BL, Mak RH, Ferrando AA, et al: MicroRNA expression profiles classify human cancers. Nature 435: 834-838, 2005.

19. Bera A, VenkataSubbaRao K, Manoharan MS, Hill P and Freeman JW: A miRNA signature of chemoresistant mesenchymal phenotype identifies novel molecular targets associated with advanced pancreatic cancer. PLoS One 9: e106343, 2014.

20. Yanaihara N, Caplen N, Bowman E, Seike M, Kumamoto K, Yi M, Stephens RM, Okamoto A, Yokota J, Tanaka T, et al: Unique microRNA molecular profiles in lung cancer diagnosis and prognosis. Cancer Cell 9: 189-198, 2006.

21. Olofsson B, Jeltsch M, Eriksson U and Alitalo K: Current biology of VEGF-B and VEGF-C. Curr Opin Biotechnol 10: 528-538, 1999.

22. Wang X, Chen X, Fang J and Yang C: Overexpression of both VEGF-A and VEGF-C in gastric cancer correlates with prognosis, and silencing of both is effective to inhibit cancer growth. Int J Clin Exp Pathol 6: 586-597, 2013.

23. Zhu P, Zhang J, Chen Q, Wang J and Wang Y: Expression of vascular endothelial growth factor- $C$ in gastric carcinoma and the effect of its antisense gene transfection on the proliferation of human gastric cancer cell line SGC-7901. Am J Surg 204: 78-83, 2012.

24. Skobe M, Hawighorst T, Jackson DG, Prevo R, Janes L, Velasco P, Riccardi L, Alitalo K, Claffey K and Detmar M: Induction of tumor lymphangiogenesis by VEGF-C promotes breast cancer metastasis. Nat Med 7: 192-198, 2001.

25. Steven AS, Achen MG, Jussila L, Baldwin ME and Alitalo K: Lymphangiogenesis and cancer metastasis. Nat Rev Cancer 2: $573-583,2002$.

26. Tammela $\mathrm{T}$ and Alitalo K: Lymphangiogenesis: Molecular mechanisms and future promise. Cell 140: 460-476, 2010.

27. Dias S, Choy M, Alitalo K and Rafii S: Vascular endothelial growth factor (VEGF)-C signaling through FLT-4 (VEGFR-3) mediates leukemic cell proliferation, survival, and resistance to chemotherapy. Blood 99: 2179-2184, 2002.

This work is licensed under a Creative Commons Attribution-NonCommercial-NoDerivatives 4.0 International (CC BY-NC-ND 4.0) License. 